\title{
Closed-loop insulin delivery has wide-ranging benefits
}

For patients with type 1 diabetes mellitus (T1DM), maintaining glycaemic control can be challenging. Artificial pancreata, which are closed-loop insulin delivery systems, monitor and detect changes in blood concentrations of glucose and deliver insulin when needed. Now, in the largest randomized study of closed-loop delivery systems so far, an artificial pancreas has shown wide-ranging benefits, including reduced mean glucose levels and reduced time spent in hypoglycaemia, in a cohort of individuals with sub-optimally controlled T1DM.

"Our [overarching] aim is to make the closed-loop technology widely available and the present study was designed to provide information to support [closed-loop insulin delivery systems] for individuals with sub-optimally [controlled]
T1DM," explains corresponding author Roman Hovorka. "We applied the standard randomized controlled trial design, included sites in two countries (UK and US) and enrolled children, adolescents and adults to increase the generalizability of the findings."

Hovorka and colleagues randomly assigned 86 individuals aged 6 years and older to receive hybrid closed-loop therapy $(n=46)$ or sensor-augmented pump therapy ( $n=40$; control group) and followed them up for 12 weeks. The primary end point was the difference between the control group and treatment group in the amount of time participants spent in the target glucose range of 3.9-10.0 $\mathrm{mmol} / \mathrm{l}$ $(70-180 \mathrm{mg} / \mathrm{dl})$, which was based on sensor-measured glucose concentrations during the 12-week free-living phase.

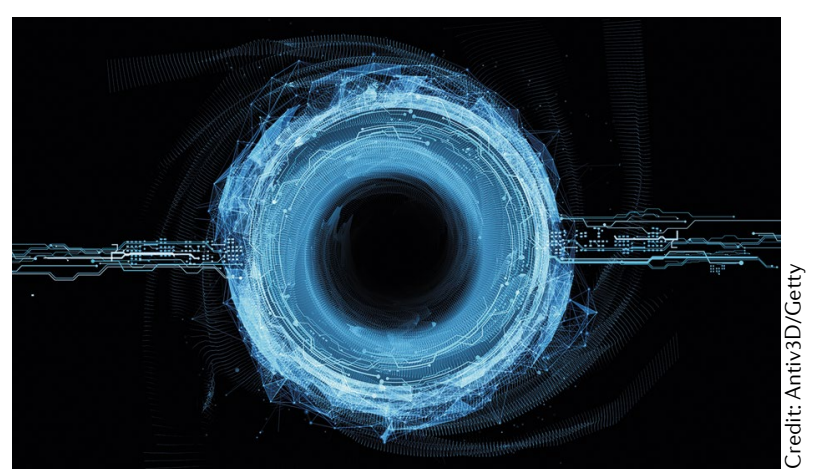

The authors report that the closed-loop system resulted in reductions in $\mathrm{HbA}_{1 \mathrm{c}}$, mean glucose levels, time spent in hypoglycaemia and glucose variability. These benefits were observed irrespective of age.

"Several studies are ongoing or are planned, including testing a closedloop insulin delivery system in newly diagnosed adolescents with T1DM, as well as young children aged 1-7 years and older adults aged $>60$ years with T1DM," concludes Hovorka. "Our ultimate aim is to commercialize our closed-loop algorithm and make it widely available."

Alan Morris in glucose variability
ORIGINAL ARTICLE Tauschmann, M. et al. Closed-loop insulin delivery in suboptimally controlled type 1 diabetes: a multicentre, 12-week randomised trial. Lancet 392, 1321-1329 (2018)

\section{Zoledronate prevents fractures in osteopenia}

Currently, the majority of fractures reported in postmenopausal women are in individuals who have osteopenia, rather than in women who have osteoporosis. Patients with osteoporosis are treated with bisphosphonates to prevent fractures; however, their efficacy in preventing fractures in patients with osteopenia is unknown. Now, new research by lan Reid and colleagues shows that treating women with osteopenia with zoledronate, a bisphosphonate used to treat osteoporosis, reduces the risk of fracture.

Reid and his team conducted a randomized, double-blind, placebo-controlled trial to test the efficacy of zoledronate in preventing fractures in postmenopausal women who have osteopenia or are on the cusp of developing osteoporosis. The authors recruited 2,000 women ( $\geq 65$ years) with a T score of -1.0 to -2.5 at either the total hip or the femoral neck and were

\section{women}

treated with zoledronate had a lower risk of nonvertebral fragility ... fractures

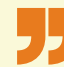

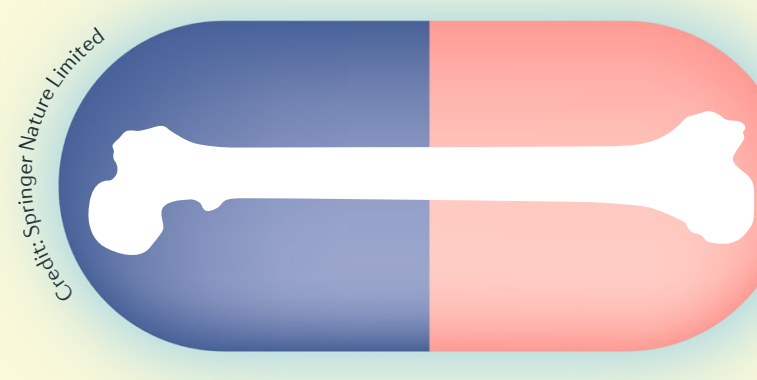

randomly assigned to either receive the treatment or placebo.

The treatment group received four intravenous injections of zoledronate at 18-month intervals, while the placebo group received saline. All participants were followed for 6 years and the primary end point was time taken for the first fragility fracture (vertebral or nonvertebral) to occur.

In the study, 190 women from the placebo group and 122 women from the treatment group developed fragility fractures (HR with zoledronate $=0.63 ; 95 \% \mathrm{Cl} 0.50-0.79$; $P<0.001)$. The authors found that women treated with zoledronate had a lower risk of nonvertebral fragility, symptomatic and vertebral fractures and loss of height compared with the placebo group.

"These findings suggest that prescribing zoledronate to a wider group of women $\geq 65$ years of age than we currently do would be worthwhile with regard to preventing fractures," concludes Reid. "The evidence that zoledronate is effective when given at 18-month intervals, together with other evidence that its duration of action extends to many years, suggests that longer interdose intervals should be assessed."

Ivone Leong

ORIGINAL ARTICLE Reid, I. R. et al. Fracture prevention with zoledronate in older women with osteopenia. N. Engl.J. Med. https://doi.org/ 10.1056/NEJMoa1808082 (2018) 